Témoigner Témoigner. Entre histoire et mémoire

Getuigen Revue pluridisciplinaire de la Fondation Auschwitz

$125 \mid 2017$

Histoire et mémoire de la persécution des

homosexuel-le's par les nazis

\title{
La SIPO-SD de Bruxelles au 347 de l'avenue Louise
}

De Louizalaan 347 en de Sipo-SD van Brussel

\section{Lieven Saerens}

\section{(2) OpenEdition}

12 Journals

Édition électronique

URL : https://journals.openedition.org/temoigner/6584

DOI : 10.4000/temoigner.6584

ISSN : 2506-6390

Traduction(s) :

De Louizalaan 347 en de Sipo-SD van Brussel - URL : https://journals.openedition.org/temoigner/ $6630[\mathrm{nl}]$

Éditeur :

Éditions du Centre d'études et de documentation Mémoire d'Auschwitz, Éditions Kimé

Édition imprimée

Date de publication : 1 octobre 2017

Pagination : 132-145

ISBN : 978-2-930953-01-4

ISSN : 2031-4183

Référence électronique

Lieven Saerens, «La SIPO-SD de Bruxelles au 347 de l'avenue Louise », Témoigner. Entre histoire et mémoire [En ligne], 125 | 2017, mis en ligne le 24 décembre 2021, consulté le 04 février 2022. URL: http://journals.openedition.org/temoigner/6584; DOI : https://doi.org/10.4000/temoigner.6584 


\section{Site mémoriel}

\section{LA SIPO-SD DE BRUXELLES AU 347 DE LAVENUE LOUISE}

Dans cet article, sera d'abord analysée la naissance, dans l'Allemagne nazie, du Reichssicherheitshauptamt (RSHA, l'Office central de la sécurité du Reich) dont dépend sous l'Occupation la Sipo-SD (Sicherheitspolizei und Sicherheitsdienst), la police SS de Bruxelles. Ensuite, seront évoquées la Belgique occupée et l'installation de la Sipo-SD. Dans la foulée, nous traiterons spécifiquement de la Sipo-SD. Dans une quatrième partie, nous nous concentrerons sur la Sipo-SD


après-guere a lencontre de la Sipo-SD de Bruxelles ainsi que des demandes en du 347 de lave Louise. Une thention particulière sera

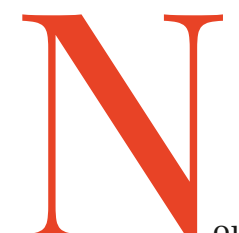

tion des Juifs (739 témoignages, dont environ 330 se rapportent à la période du 347 delavenue Louise) ${ }^{1}$,'arrestation detions individuelles; des tém d'arresde résistants arrêtés en throupe, cong de résistants arrêtés en groupe, comme ceux dune cinquantaine de Partisan. les més capturés en 1944), lenquête su les ménbres allénands de la Sipo-SD la structure de la Sipo-SD. Signic) et la structurute la Sipo-SD. Sur ce plan, Les renseigne les Archives Générales du Royaume les Archives Generales du Royaume concernant les Dommages de guerre totalement inédites.
LE REICHSSICHERHEITSHAUPTAMT Le 27 septembre 1939, après l'attaque de la Pologne, le Reichsfuhrer-SS etrich siche sicherits (Schutzstaffen, section de protection du parti nazi). de protection du parti nazi). Le RSHA des diverses instance dellemeau de e service central de renseignent et de ścuré du Reich. Il est place et de sécurite du Reich. Il est placé drich, jusqu dienst (SD, service de sicurtén dienst (SD, service de sécurite) et de la Geheime St l'spolizei (Gestapo, police gruppenführer . En 1 Kalle SS-Obergruppenfuhrer Ernst Kaltenbrunner de police à Theydrich. Tres ses Reichssicherheitshauptamt ${ }^{2}$ :

Le Sicherheitsdienst (SD, un service de renseignement idéologique et politique ouvrant pour les SS et le parti national-socialiste, qui a pou but de protéger le parti nationalacialiste contre ses ennernis, soit à ses yeux les marxistes, les socialistes, les liberaux, les Juifs et La Geheime Statspolizei (Ge

La Gehein

Julizol police judiciaire, un organe d'investigation existe depuis longtemp.

Auparavant, en 1936, la $S D$ et la Gestapo avaient déjà fusionné sous le nom tapo avaient deja fusionne sous le nom politique proprent (Site de lallepolitiue proprement dite de lAlle-
LA BELGIQUE SOUS LOCCCUPATION ALLEMANDE

Après sa capitulation, la Belgique allemande (Milituristration militaire cénéral Alexinur vollk), avecle com (aber) et la re de von Falken ruusen dirige une Servaltungstab (cellule de des affair (celluite in

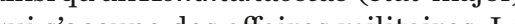

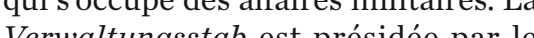
Militärverwaltungschef Egrect par le le Komman tab par lobert Reeder, Bodo von Harbou4.

L'administration militaire disose de services de police militaires : pose de services de police militaires lorde (GFP, police secrète mita (GP , police secrète militaire). La FG dastab fe Bodo partie de la Komman-

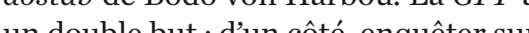
les méfaits commis par et coêter sur militaires allemands ; de lavie les contre les éléments contre les elennents choma a a Résistance, les citoyens juifs. Pour léchange d'informations, lu Feldpolizei collabore avec l'Abwehr. Dans lize collabore avec l'Abwehr. Belges - tant Juifs que nonétes sur des tissen - très vite à que noońts - aboula Feldgendarmerie, la Geheime Feldpolizei et la Sipo-SD. En outro il existe lizei et la Sipo-SD. En outre, il existe Polizei, qui polève du Verwatrungsstab d'Eggert Reeder.

Au cours de la première année de Oadministration militaire est soumise à la concurrence de

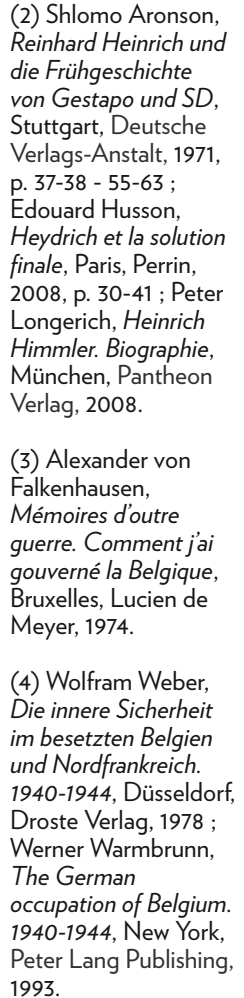


• la police SS allemande, composée de représentants du Reichssicherheitshauptamt (RSHA). Début juillet 1940, tor Fran Strab, sculpteur de formator Franz Straub, sculpteur de formad'environ à Brist sipo denviran 20 agents Sipo-SD. Legroupe Sonderkit (GFP). Bien queles agents Feldpolize (GTP. Bien queles agentsportentlunicor e $R S H A$

Le 27 juillet 1940, le chef du Reichssicherheitsheithauptamt Reinhard Heydrich se rend à Bruxelles pour l'inauguration officielle de la centrale Sipo-SD, installée après l'approbation de lOberhommando des Heeres (OKH), leconnandementenches des forces terre allema de et chef de la police Heinrich Himmle nomme un seul representant pour Beauftragte des Chefs der Sipo und des Sicherhetsdiensts für Belgien und des Sicherhetsiensts fur Belgien und Franfïhrer dr Tax The par le SS-Brigadefuhrer dr. Max Thomas, un psychiatre Thomas dispose de deux Dienststelle (sièges), nà Paris et lautreà Bruxelles. celuide Braxelles est, commeladministration militaire, égalomentcompétent pour le nor de la France (Nord et Pasde-Calais). A partir de déccorbre 1941,

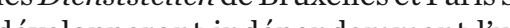
developperont indenconment au Reichssicherheitshaumptamt.

a Sipo-SD,composée de membre de la Kripo, du $S D$ et de la Gestapo, doit, politique du ntioe, «assurer lordre politique du national-socialisme alle- l'unité comporte six Abteilungen (sections), elles-mêmes subdivisées en domaines et services. et II se chargent, en principe, de l'adminitran la section I soc upe avant cont la section II soccupe avant tout de la persécution des Juifs et des enquetes ratives aux activités de la francniçonnere et dos « Égises «. Lorgaprís

A. Section I : Administration

B. Section II : Affaires juives, francmaçonnerie et ecclésiastiques.

C. Section III (Sicherheitsdienst) Espionnage; par la suite, elle s'occupera aussi des réfractaires au travail

Dection IV (Gestapo) : Agitation communiste et socialiste. Agitation des mouvements de droite et libéraux. Etrangers : passeports - rapatriement en Belgique - recensement Fichier général.

. Section V (Kripo) : Affaires criminelles, c'est-à-dire vols, recherches

F. Section VI : Nationalité et espionnage commercial.

Les arrestations et les rafles organisées par la Sipo-SD se déroulent génésees par la Sipo-SD se derroulent génémenées par nuit et sont en principe

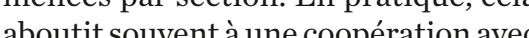
des memones d'un cooperation avec des menbres dune autre section. Pendant les actions de grande envergure, au cours desquelles un groupe entier gées, à côté de tous les mentassiengaSipo-SD, la Geheime Feldpolizei et la Feldgendarmerie. Cette collazizi et la restégande. Cette collaboration rafles de Juifs. La Sipo-SD est tristement célèbre pour ses «interrogatoires renforcés» et les mauvais traitements, physiques et psychologiques. On reviendra nant les process d'apris-guerre et les ám les procès témoignages.

LA SIPO-SD À BRUXELLES

Lepremierchefdu postedeBruxelle est le SS-Sturmbannführer (major) und Oberregierungsrat Karl Hasselbacher, un docteur en droit. Après sa mort le de voiture, il est roms un accident Sturmbanffïhrer und Oberre par le SSStur ench endroitethistorien. Ennovenore 1941, Oberregierungsrat Ernst Ehlers médecin qui en 1940 fait Lhers, un éff des

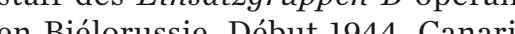
reprend la direction du poste.

Au total, durant toute l'Occupation, Au total, durant toutel'Occupation,
au moins 550 Allemands travaillen au moins 550 Allemands travaillent pour la Sipo-SD de Bruxelles. Ils sont au 64 dans 127 dion 216 dan I tion IV,61 dection II, 216 dans la sec. ja jutice d'après-guerre n'a pu découla justice daprès-guerre n'a pu découćm sément servi. De ces chiffres, il apparait clairenter que la section IV (Gestapo) est la plus fournie en personnel et que par consequent, elleétait als conside re conne très inportante.

Oulaborateurs belges font également partie d Wachug (Corps SD. un Wachzug (Corps de garde) de la Sipotué en grande partie de SS flamits, comme l'Anversois Lode Van Kol. Au total, 25 Allemands au moins font partie du Corps de garde au cours de l'Occupation. Les nen bres allente pont a ditiśs Cosin sont déportés) et à Breendonk. Par la ( (canons antiaériens), dont des canons ont installés au-dessus des bâtiments de Sipo-SD. Parsi eux, I'A Verbert.

Des collaborateurs sont aussi liés aux différentes sections de la Sipo-SD mme traducteurs-interprètes et embres de la SS. Ce sont, pour la plu, des Fanands, commel'antisémite Jul ul. Enfin, il y a encore des V-Männer, des «hommes de confiance» qui trafaillent pour la Sipo-SD. Parmi eux figurent Prosper De Zitter de FlandreThion

Men (alya Maxiptinthe

Mais il y a aussi pas mal de dénoncia(eurs juifs, comme Icek Glogowski («Le Gros Jacques "). Si «Le Gros Jacques» pans les milieux contians, il nest lans les milieux concernés, il n'est pas ent Juif à dénoncer ses coreligiondizaines, il sogit sou à lépoque par de réfugiés

Le quartier général de la Sipo-SD de Bruxelles est d'abord établi à l'Hôtel des Croisades à la Gare du Nord, puis à l'avenue des Tilleuls (à Uccle, dans la maison d'un sénateur qui a décampé), un peu plus tard avenue Ernestine $n^{\circ} 2$

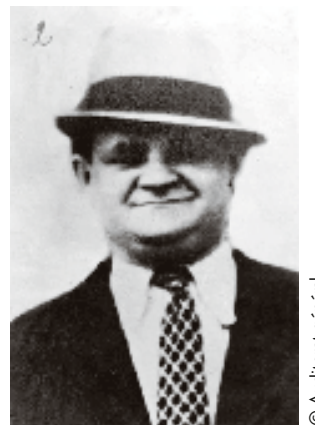

I Iceck Glogowski, alias «Le Gros

Jacques"

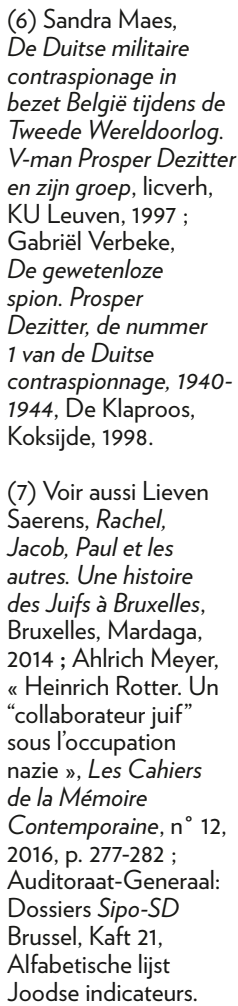


(8) Guido Provoost, Ward
Hermans, Anvers, Rockox,
1977, 153 .

(9) Archives générales du
Royaume (dépôt Cuvelier) Dommages de guerre,
Dossier 20.28.739, Georges (10) Archives de la Ville de Bo Archives de la Víle de
Buxelles: Dossier réquisi-
tion allemande; ${ }^{\circ} 4663$.
• Sipo-SD occupe les bâtiments portant es numéros 372,453 et 510 . Les services des sections I et II sont au départ (Résidence Belvédè 410 . Le n ${ }^{\circ} 45$ (Résidence Belvédère) héberge aussi à lorigine la section IV, tandis qu'au dant toute l'Occupation la section dant toute loccupation la section et une partie du parc automobile. Un aut 518 de la même av-SD situe au '5 la la me la dans lavenue Louise, sont hébergés pendant toute loccupation au n 372 la section III, ainsi Outre

Outre la Sipo-SD, d'autres service delass allemande sont installés avenue Louise. Ainsi, depuis le 1 é avril 1942 est établi au no 412 le poste Jungclaus, dirger par le Huncre SS und Polizeijüdirection Jffective de toutespose de drection Jitive de tou les onansaSS, pontre autres les SS-Vlacanderen, la Duitsch-Vlaamsche Arbeidsgen, la Duitsch-Vlan msche Arbeidsgeneenschap (DeVlag), (le siège de Rex est égade 1944). Ilesten mêm adesse à partir de 1944). Hest en ment temps responSS (com rattants sur le fes l' s. (combars

rateurs militaires belges).

\section{LE 347 DE LAVENUE LOUISE}

Le 20 janvier 1943 a lieu le fameux raid du pilote belge de la $R A F$ Jean de Selys Longchamps sur le quartier général de la Sipo-SD, au numéro $453 \mathrm{de}$ le batine Louise. Après avoir mitraille le bâtiment, il jette deux bombes de 250 kilos sur ce dernier, détruisant Cinq membres de la Sipo-SD Cinq l'ate cection UI, Alfed Thonas. Ily a du quatre blessés graves.
Suite au raid, ce bâtiment est perdu pour la Sipo-SD qui occupe à partir de pour la Sipo-SD qui occupe a partir de de l'avenas 1943 le bâtinent du 34. ine par gine, parmilesquels dailleurs plusieurs des ancetres juifs, ju l'Ortsher surs, sont Ortshomar locind

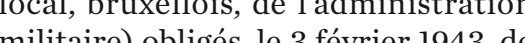

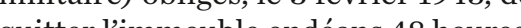
Litter les déménageurs sont imposés pa

La 6 fevrier 1943. Ce jour-là, les habitants du 347 de lavenue Louise font faire par lexpert Servais une descripcet imm is à app les partants de cet ine tions pere inportante : ces descriptions permettent, après la guerre, de déĉsts cégâts occasionnes par la Sipo-SD et des

Apparimment la Sipo-SD a camméApparemment la Sipo-SD a emmésitué au 347 de l'avenue Loun sévie au 347 de lavenue Louise le 15 pris quelques semaines pour te encore

Dorénavant l'organigramme de la Sipo-SD se présente comme suit

A. Section I : Organisation, Affaires de personnel, budgétaires et économiques (sous la direction du SS-

Hauptsturmfuhrer Hofmeister).

B. Section II: Appointements, frais de voyage; Caisse; Logements, besoin. du service, habillement, avoirs salsis, etc. ; Ravitaillement ; T.S.F. Télegraphie et Teléphonie; Service irection du SS-Obersturmführer Lüdtke).

C. Section III : Vie publique (Droit, Constitution, Législation, Administration, etc.; Vie nationale et santé publique ; Vie intellectuelle ; Économire nationale, Sous la direction usS-Sturmbannfuhre

D. Section IV Gestapo : Recherche et répression des adversaires (Communisme et marxisme, falsification de passeports, propagande ennemie tiques; Franc- Lanse et sectes politiqfes ; Trancpases dent et falsification de passeports et laiset falsification de passeports et laissez-passer sy rapportant; Répresbendes; Fichiers, Dossiers, Détentio pévictionprère; Secréta SS-Sturmbannführer Franz Strab.

- Sestion V : Répression de crines

E. Section $V$ : Repression de crimes (Affaires : Rérales de Police crimde tion; races ; Service didentification, Laboratoire de criminologie Secretariat) sous la direction du SS-

F. Section VI : Affaires de renseignementsouser lirectionduSS-Obers-

$\mathrm{Au} 347$ de l'avenue Louise sont hébergés (au minimum) les services suivants :

- Section I

Section IV (Gestapo)

Section VI

Les plus petites sections I et VI s'installent probablement au $1^{\text {er }}$ étage.

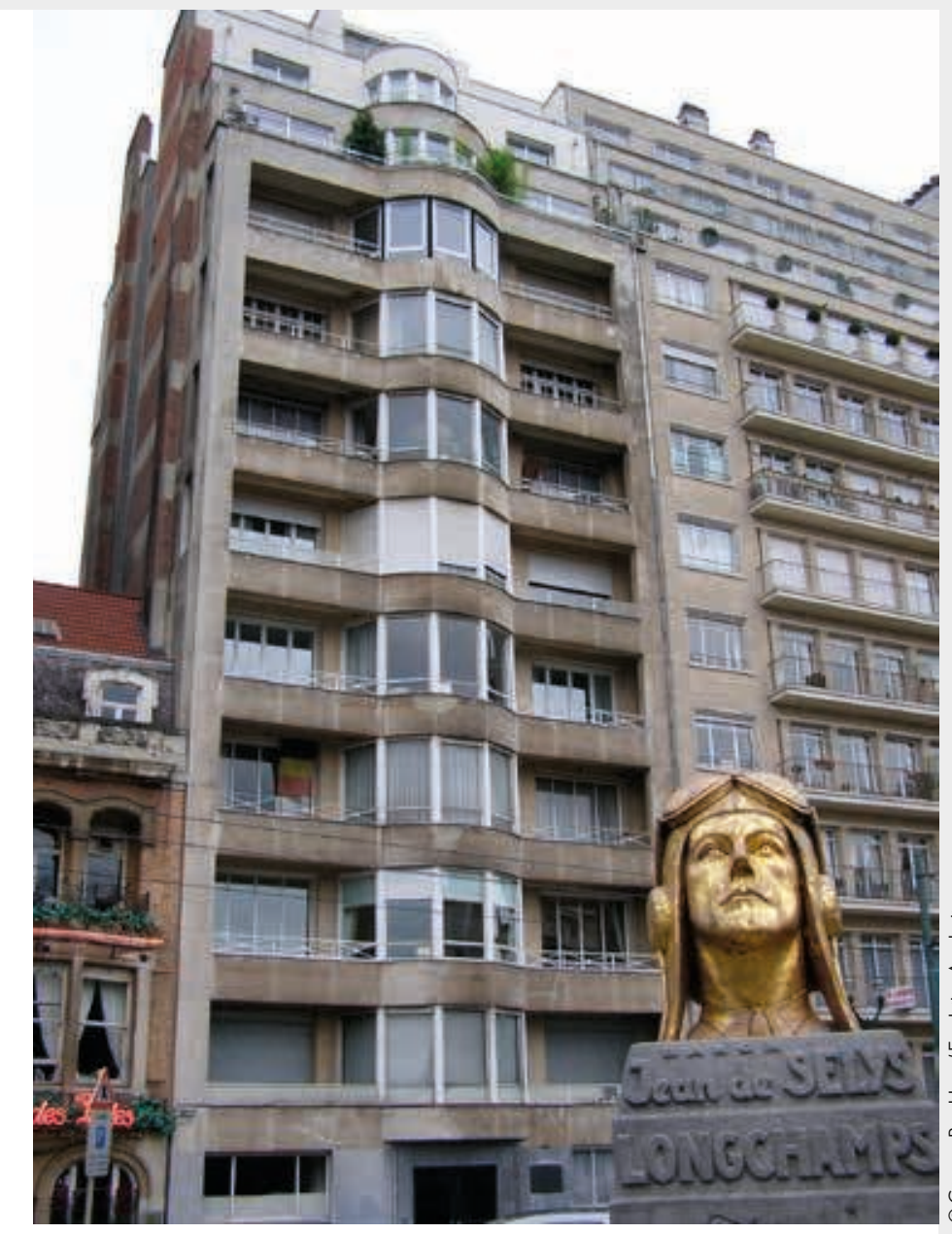

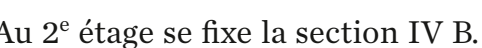
453 avenue Louise à clle-ci se compose de trois sous-sections :

- Section IV B 1 : Églises et sectes politiques, sous la direction du SS-

- Section IV B 2 Franc- Seck.

- Section IV B 2. Franc-maçonnerie, führer Fritz Erdman-HauptsturmJuhrer Titz Bid 3 : Jifs,

- Section IV B $:$ Juifs, sous la direcWeidmann. 
Aux $3^{\mathrm{e}}$ et $4^{\mathrm{e}}$ étages sétablit la section IV E (Fichiers, Dossiers, Détention préventive et affaires de Légion étrangère), Wilhelm De til, que se situe le bureau decte Gestapo, Franz Straub.

Aux $5^{\mathrm{e}}$ et $6^{\mathrm{e}}$ étages prend place section IV D (Répression d'organisme section IV D (Repression dorganismes tion du SS-Hauptsturmführer Heinz

$\checkmark$ Plan des caves 9 de l'avenu

Volkmann.
Les $7^{\mathrm{e}}, 8^{\mathrm{e}}$ et $9^{\mathrm{e}}$ étages sont investis par la section IV A (Communisme et marxisme far propagande ennemie et Police belge).

Aux $7^{e}$ et $8^{e}$ étages est aussi installée Commission spéciale qui était aupala Commission spéciale, qui était auparavant établie rue de l'Aurore. Elle a été nise sur pled par Franz Straub, le chef de la sectionIV (Gestapo) et a été creée pour Sipo-SD aveche la coperation de la d'attentats commis contrela Wehrmacht.



Le $10^{e}$ étage est occupé par la section IV C (il est possible qu'elle occupe aussi une partie

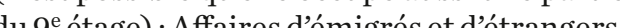
ches in fication de passeports et laisser-passers rapportan

À l’arrivée des Alliés, le 2 septembre 1944, la Sipo-SD abandonne le bâtiment du 347 de l'avenue Lovise Sur laccotemt du Jardin du Ro nue Louise. Sur laccotenent du Jardin du Roi sier Après la Libération, un quare un prénbritannique y est installé pu quatier genéral temps, plus précisément pu 16 septer

APRÈS-GUERRE : LA POURSUITE PAR

LAUDITORAT GÉNÉRAL DE LA SECTION IV DE LA SIPO-SD

Les procès belges contre des criminels de guerre allemands ne commencent que for tard.ilfaut lesidentifer, leslociliseretles faire extrader, ce qui prend énorménent de temps. se dote d'une loi autouls se dote dune loi autori ju des ét angers.

Selon le dossier judiciaire d'après-guerre de l'auditorat genéral, les méthodes utilisées par la Sipo-SD enfreignent clairement «les lois et pratiques de la guerre». Les «traitements et méthodesn utilisès sont «criminels». Pour questionner les prisonniers, on va jusqua " «interrogatoire renforcé», ce qui est un minimum eur déte et à des blessures lors de l' interrogator

Par ailleurs, une enquête est ouverte au sujet des activités de la Sipo-SD de Bruxelles, sujet des activités de la Sipo-SD de Bruxelles, Buxelles Comme cest le cas par Bruxelles». Comme cest le cas par exemple pour Anvers, lenquette se linite finalement à la section IV de la Sipo-SD, la Gestapo, «cette ment la section IV A. La toute grosse majorité des 543 membres allemands de la Sipo-SD de Bruxelles dont la justice connaît les nom appartient à la justice ouvre une enquête sur 66 d'ente eux. Dix sontcondamnés, dontlo Dix sonvelles Constantin Caco de Bruxelles Constantin Canaris. Un autre chef

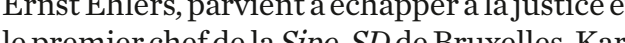
Hasselbacher, décède au début de la guerre. Sept descher 347 de l'avd Louse Outre le ch de la tion IV Franz Stris. Ontre le chef de la section IV, Franz Straub, celaconcerne le Krimina Sekretar Emiel Spete, le Kriminal Angeste Sehretar Emiel Spaete, le Kriminal Angestelte Her Alch Cuypers et le Kring Sehretar WalBoden (IV B), Otto Siegbrit (IV B et IV C) Boden (IV B), Otto Sieghang (IV B et IV C) et libérés vers 1951.

Élément non moins important : la justice militaire ne peut poursuivre les membres de militaire ne peut poursuivre les nembres de sous l'application de l'un ou l'aut are ande notre Code pén nation oul C'est poure hos membres de la Sipo-SD ne sont poursuivis que pour les conséquences in poures par leurs méthodes ; pour les coups et blessures occasionnés par les interrogatoires renforcés.»Cela ne signifie pas que l'auditorat géné ral ne travaille pas de manière approfondie. recin des anc des anciens prisonniess déction IV: d'une part, les Juifs. Pour chaque groupe, un modèle de questionnaire est établi où l'avenue Lodel est explicitement évoquée et l'arrêté(e) doit est explicitement évoquée et larreté(e) doit pévices subis. Le questionnaire semmissaires de police et aux commaye aux de gendarnerie de toutle pays. darmerie de tout le pays.

Des quelques milliers de témoignage recueillis par la justice d'après-guerre, il $\bullet . \bullet$ 
• apparaît incontestablement que les caves du 347 de l'avenue Louise on été transformées encellules et qu'elles ont res. turs. Certains prison iers en sont (uen uncenter Belge Antoine Deaunerie (Ostende, 20 mai inis) explique à J. Warnant

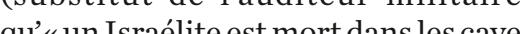
du juin 1943 (a ditortgén Sipo-SD de Brutelles, carton 12) Sipo-SD de Braxelles, carton 12) Il sag iciplus précisénent du Juif Hilel Erner, lid ans au moment des faits, arrêté par jullemand Otto Siegburg de la section juive (section IVB). Erner sttellement maltraité par Sibghirg quil décede de après avoir été roué de coups ${ }^{12}$

Un partisan, Emile Meerlandt, ne survit pas, début avril 1944, à so interrogatoire au $9^{\circ}$ etage. La Sipo-SD affire quil s'est suicidé en sautant par la fenetre. Le rapport dexpertise nediautre conclusion

Il nous paraît difficile d'accepter l'affirtetion de la police allemande selo laquelle Meerlandt aurait sauté du 9 étage, soit dune hauteur d'enviro 40 mètres. Les fractures du crâne, les fractures du bras gauche et des deux avant-b́ peuvent naturelloment être laconsén parait anonalo cest quinechute dune ture de ture sootes, de lacolon dubsint des nem été la victime de violen les fractures du crâne et des membres supérieurs auraient pu être la conséquence.

(Rapport autopsie Meerlandt par les docteurs Marcel Heger et Paul Enderlée, requis par lauditorat militaire

Mentionnons aussi l'arrestation, au intemps 1943 , printemps 1943, des chefs des Partisan le bâtimén dunt ensulte amenés dan e batiment de la Gestapo à Bruxelles fuits a Louses de faits tel qu il est établi apres-guerre elles, il est indiqué :

Pour ce qui a trait aux mauvais traitements subis dans le cadre de cette affaire ll convient d'attirer particulièrement lattention surle fait que les femmes, qui, ans cette affaire, avaient été soumises à des tortures, sont restées pendant des jours et monne des semaines enfermées dans les caves de la Sipo dans des conditions indescriptibles. Ce n'est que lorsque les traces de violence avaient portées à la pro ques furt-Gilles. [.] portes à la prison de Saint-Gilles. [...] Messieurs Devoitille, Jospa et Vandeville ont contracté une incapacité de travail permanente. La bronchite chronique trousthe de la ténoin Korn Adele trouvent leur origine dans lenfermant d'hysiène d'un fordes manquant d hygiène dune fenme qui auparavant avait été rouée de coups su
tout son corps ${ }^{13}$.

La prison de Saint-Gilles constituait selon toute vraisemblance une solution de rechange quand les cellules de la Sipo-SD du 347 de l'avenue Louise faits ne laisse p. Ce même exposé de le fait quisse planer aucun doute sur la Gestapo a appliqué l'«interrogatoire renforce» dans ses locaux (et donc aus au 347 de lavenue Louise). A ce sujet, est notan

de limites [àl'interrogatoire renforcé], il n'en était pas question et encore moin de lintervention dun medocind dans les cas graves. Il n'est que trop évident que la Résistance et pour y a ive toun la Resistance et pour y ariver, tous

Nous disposons également de la déclaration d'après-guerre de Denis Caenen, qui affirme avoir été membre des Milices patiotiques du Front de Gestapendéce. Dans les bureaux de Gestapo, déclare-telle, jai subidivers rerdu toutes mes dents suiteaux coups

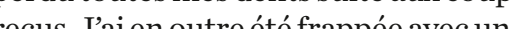
reçus. Jai en outre été frappée avec une matraque sur tout le corps. Ces mauvais triante ondant trois jours ter en tr concentration juin 1944 au canp de concentration pour fenmes de Ravensbrück, en Allemagne. «D fait de ma captivité et des mauvais trai$50 \%$ Le 23 avil 1945, elle tibe , $50 \%$ Le 23 äl 1945 , elle est libéree de Ravensbruck et rapatriée vers la en Belgique ${ }^{15}$ en Belgique ${ }^{15}$.

La Juive Juda Rozenberg, elle aussi «subi des sévices, c'est-à-dire des coups de poing» (Ibid.). Ernest Abel, négociant en laine juif, témoigne de a section juive

Erdmann m’a frappé au front et au cui chevelu, me provoquant une blessure à sang coulant, il m’a aussi porté plusieurs coups de fouet. [...] J'étais battu jusquà évanouissement.

George Beigne, arrêté dans la région bruxelloise dans la nuit du 15 au 6 mai 1944, est interrogé à plusieurs reprises au 347 de l'avenue Louise :

J'ai été battu à coups de matraque au point que durant quinze jours j’ai dû dormir. Un moment doné au couvoir dormir. Un moment donné au cours de cesinté gatorres, les Allemands mont Jurncéla tête dansun bassin plein deau jusqua ce que je perde connaissance. été de mon du été suspendu les bras deribre le dos et battu dans cette position.

Le 15 avril 1944, l'instituteur de 8 ans Lodewijk Verheyen est arrêté à Buizingen (Halle) Jlestim tidentét emmené au 347 de l'avenue Louise, où lint bourré de coups et frappé sur mes parties intimes. La Juive sace . du rond-point du boulevard du Souverain à Auderghem, est interrogée $q^{e}$ étage et raconte: « [Un hommel avait mis sa veste sur ma figure pou avan oute étouffer mes cris. J'ai été san de coups au point que je ne savais plus m’asseoir.»

Ces mauvais traitements laissent es traces dans les caves du 347 de l'avenue Louise, comme le signale Nelly Pardons : «Je puis vous dire qu’on m’a bbligée le jour de mon arrestation à Aettoyer les cellules à lavenue Louise. Alusieurs plusieurs cellules il y avait du sang sur 
$\because \quad$ Les DeMANDES DE DÉdomMAgement ET LES INSCRIPTIONS DANS LES CAVES

Que les caves aient effectivement été

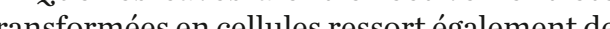
manièrévidente des dossiers Donent de guerre ên troduits après-guerre aures de gueren introduts après guerre aupres du publics et de la Recostruse des publics et de la Recóntroction) par les habitants et/ou propriétaires du 347 de lavenue Louise. Tous les dossiers nont pas été conserrés en entier, hais du fichier tel quil a pu être retroun rue du Houblon à Bruxelles (dépôt Cuvelier), il apparaît que tous les habitants du 347 de lavenue Loulse ont introduit après la guerre une demande de dedonilagement. Des géodresé exp procès-verbal d'état des pripe, dressé unprocès-verbal détat deslieux détaillé. Les dégâts imputables aux Allemands y sont des caves y est aussi chaque fois donnée.

Mentionnons ainsi le dossier de Jacques Levy-Morelle (propriétaire des appartements
des $5^{\circ}$ et $6^{e}$ étages), établi par A. Edouard Adam et Fernand Coenraet, « tous deux géomètres experts immobiliers, légalement admis et assermentés par-devantle Tribunal de Première Instance séant à Bruxelles.» Il porte comme titre «Procès-verbal d'État de Lieu [Bruxelles, le 3 et jours suivants mars 1946] - Dégradations allejours suivants mars 1946] - Dégradations allede la prise de possession du batine nom de la prise de possession du bâtiment par les durand 646 for 19.3. Les pages 35 à 37 détaillée de la sitution destunedescription $\sigma^{e}$ étage : au $6^{\text {e étage }}$

Première cave : elle se trouve la première à front de rue et vers le $\mathrm{n}^{\circ} 345$

nensions $4,90 \mathrm{~m} \times 3,00 \times 3,00$

Le

fond, les mursetles caveaxest fottenent souillé et poussiéreux.
425. La porte à claire-voie séparant la cave a été enlevée

[… métallique.

428. Le panneau supérieur de cette porte a été découpé pour permettre la pose d'un judas. [...]

431. La peinture de la porte est complètement dégradée par la pose des tôles et d'inscription diverses.

Note : Le pavement de cette cave est fortement souillé. Cette cave a servi comme cachot.

Deuxième cave (contigüe à la précédente)

\section{$4,90 \mathrm{~m} \times 1,60 \times 3,00$}

432. Le badigeon du plafond et des murs est fortes é dégradé par des inscriptions. 433. Laporte decette cave aété également recou-

434. Le panneau supérieur de cette porte a été découpé pour permettre la pose d'un judas.



Pour la cave appartenant au $5^{\mathrm{e}}$ étage, la description est presque identique. Le dossier cite et De Backer (4 étage) est tout aussi expliment sovilé rempli d'inseriptions

Cinquante ans plus tard, lors d'une aprèsmidi d'octobre 1994, le cinéaste et historien Andre Dartevelle redecouvre les inscriptions. Cette découverte déboun cat Gestap. In de helders van de la Gestapo. In de helders van de Gestapo, un ouvrage publié par le Centre de recherche et detudes historiques de la Seconde Guerre martiale (lactuelCegesoma) etrédigétant par teville. Trente par lhistorienne Isabelle Pontans. Trente photos des inscriptions visibles dans les caves de l'avenue Louise sont publiee
Les inscriptions sont incisées dans la couche de chaux et non dans la brique, à laide de petits instruments métalliques, pointes de plumes, épingles, attaches cousues dans les vêtements,

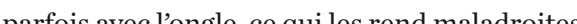
Les détenc liont laí sage ont parfois écrit leur messase ou leur nom

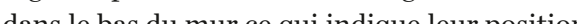
assise ouc corche a mêne le La plpat des greffitisontén Plus rarement le bras tendu (p. 42).

En 2011, Sarah Timperman, archiviste à la Fondation Auschwitz, retrouve fortuitement Fondation Auschwitz, retrouve fortuitement dans les archives du Musee royal de l'Armée (Bruxelles) les cénéral prises au moment de la Libération en genéral prises au moment de la Liberation en septembre 1944 par les soldats canadiens. Son collégue, lhistorien de l'art Daniel Weyssow, présente ces photos lors de la journée détude Les caves de la Gestapo. Recounass 2011 pe conservation organiscé le Fondation Auschwitz

Enfin, à la demande du Conseil de Copropriété de l'immeuble Queen situé au 347 de l'avenue Louise, une visite des caves est organisée le $1^{\text {er }}$ juin 2015 pour constater de visu lexistence ou non de graffitis sur les murs des meuble. Sont présents lors de cette l'auteur de ces lignes et son collègu Fabrice Maerten, Roland Cracco, un des propriétaires de l'immeuble, Stéphane Nopère, sieurs personnes travaillant pouriété, et plusieurs personnes traillant pour la Direction l'A Ministrent, du Ministerre de la Région de Bruxel arry Lelièvre.

Lors de cette visite, 24 caves sur 26 sont accessibles. Si aucune inscription n'est visible dans les locaux techniques, dans les couloirs, et dan certaines caves individuelles, des graffiti datan avec quasi-certitude de la période 1943-1944 son repérés (parfoisfacilement, parfoisdifficilement), avecl'aide des délégués de la Direction des Monuments et Sites, dans 9 caves individuelles.

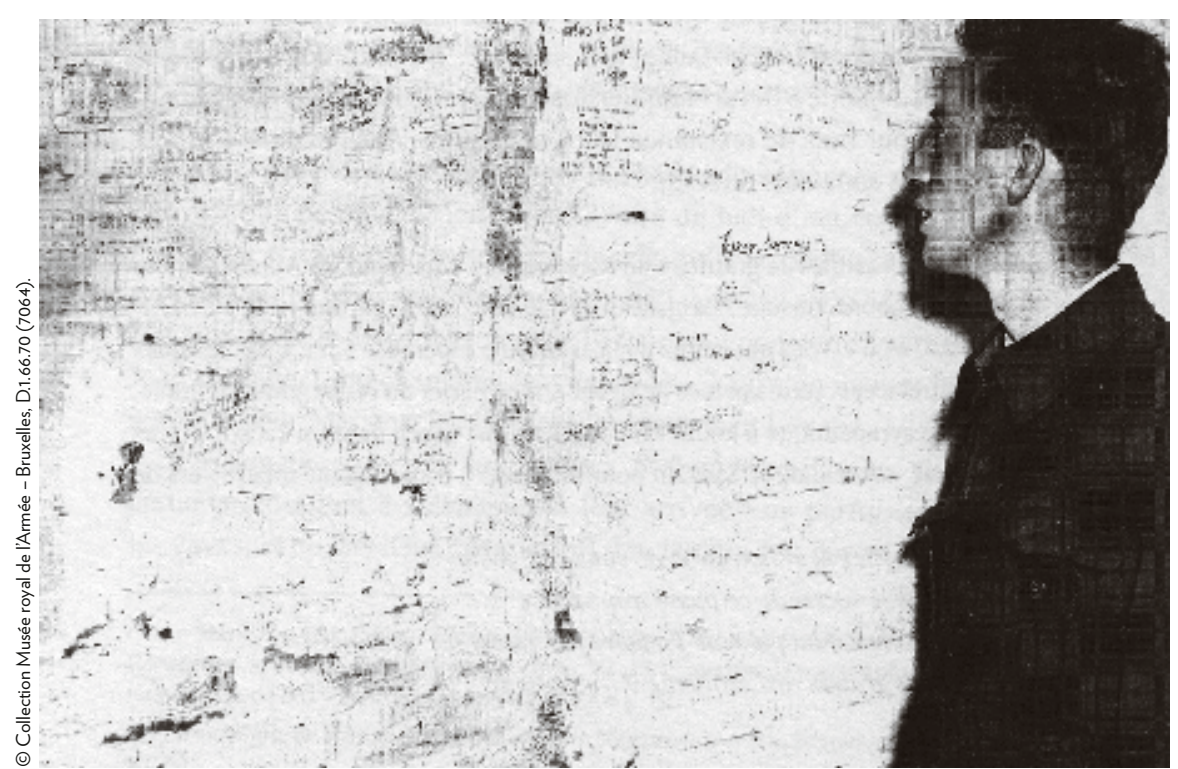

$\forall$ Messages écrits par les prisonniers sur le mur d'une
cellule au 347. 


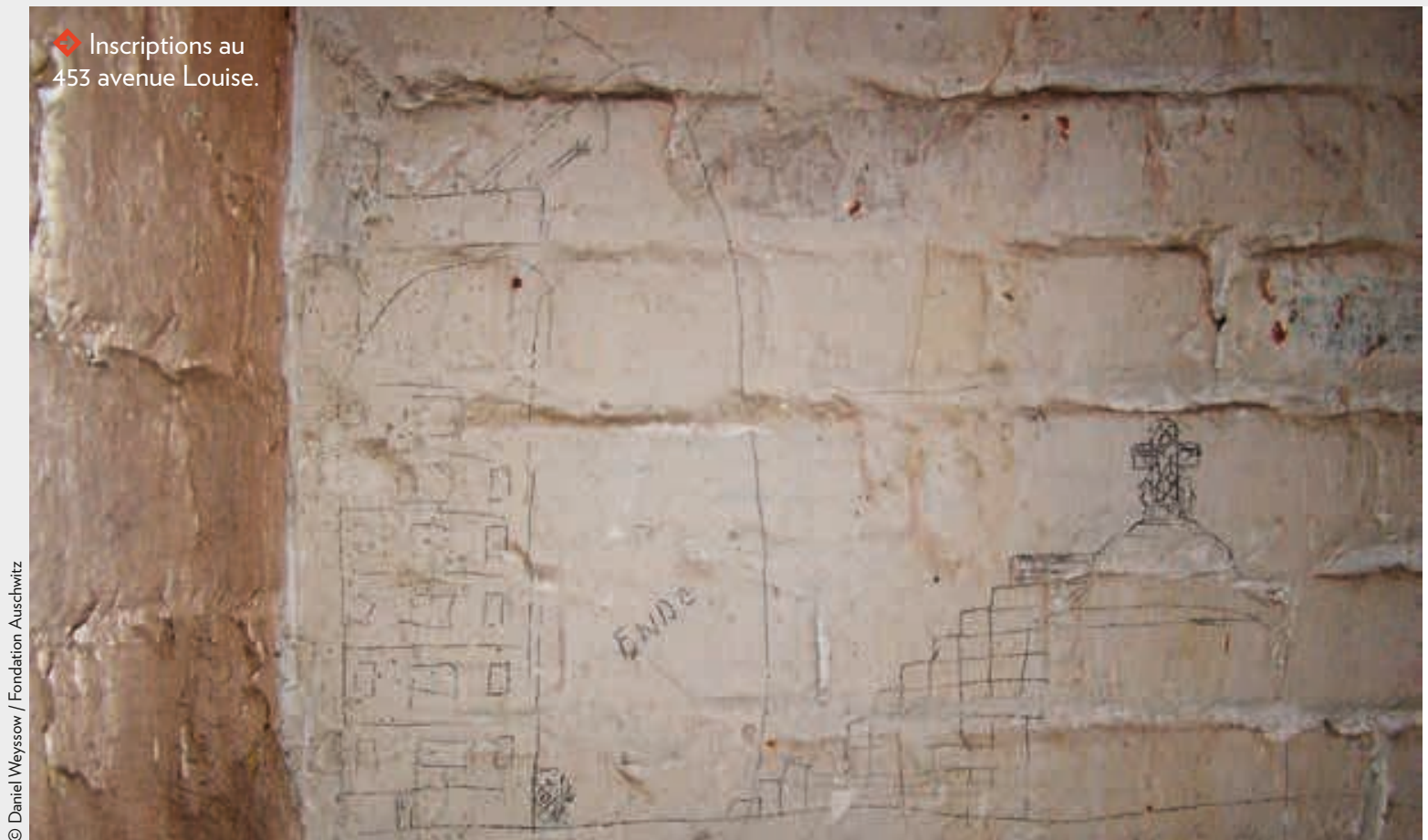

CONCLUSION

Après le bombardement de Jean de Selys Longchamps des locaux Sipo-SD au 453 de l’avenue Louise, le service s'installe dans les appartements du 347. Les caves de l'immeuble seront bientôt transformées en cellules où les détenus, des résistants et des Juifs, sont frappés a sang et tortures, comme le soulignen de nombreux témoignages récoltès dans límmédiat après-guerre dans le cadre de lenquête judiciaire contre les membres allemands de la Sipo-SD de Bruxelles. Pour ressister à cette inhumanité, de nombreux détenus ont laissé des inscriptions sur les murs, les plafonds et les portes des caves. Certaines sont encore visibles en 2015 , dautres pourraient être retrouvées sous le badigeon. In ne fait dès lors aucun doute pour nous quéces caves constituent unimportant «site» historique quiliconvient de préserve En jo eséver

Le siège de la Sipo-SD à Anvers au Della Faillelaan (août 1940-octobre 1943) existe toujours. En 1967 on y installe le siège de l'évêché d'Anvers, la villa appartient désormais à une personne privée D'après le défunt aussi ce lieu de mémoire?

Je remercie Fabrice Maerten pour la traduction et la relecture scientifique.

\section{$\rightarrow$ Sources}

Archives Générales du Royaume (Dépôt Cuvelier)

$\checkmark$ Auditorat général : Dossiers Sipo-SD de Bruxelles (Straub et consorts : Vits, spaete, Cuypers, Altenhof). 28 cartons. $\diamond$ Auditorat général : Dossier Felix Weidmann (chef de la section juive de la section IV. Ce dossier contient aussi des PV relatifs au chef de la Sipo-SD de Bruxelles Ernst Ehlers
et à d'autres chefs de la section juive comme Kurt Asche et et à d'autres chefs de la setr.
Fritz Erdmann). 1 carton.

$\diamond$ Auditorat général : Dossier Emiel Francken (interprète de

$\diamond$ Auditorat général : Dossier Reggie Claeys (interprète de la section IV). 1 carton.

$\diamond$ Auditorat général : Dossier Otto Siegburg (section IV). 1 carton.

$\triangle$ Dommages de Guerre : Dossiers n's 20.28 .739 (Georges Fleurix); 20.77 .252 (J. Levy-Morelle); 20.89 .621 (Mme Lucien Niguet) ; 20.89.623 (Guillaume Wallenborn); 20.89 .625 (Entreprises Edouard François); 20.89 .627 (veuve 20.89.631 (Mary Partas) : 20.89 .633 (Charles Sohal) 20.89.635 (Mme Jules De Backer); 20.89.637 et 23.39.41823.39.424 (J. Levy-Morelle) ; 23.39.455-23.39.457 (veuve Lucien Niguet).

\section{Archives Service des Victimes de la Guerre}

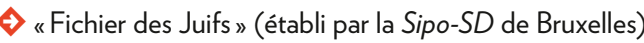

$\diamond$ Dossiers Sipo-SD (n ${ }^{\circ}$ Tr. 202.411 - R. 497).

\section{Archives CegeSoma}

$\triangle$ Procès Constantin Canaris (chef de la Sipo-SD de

$\Leftrightarrow$ Archives Pierre Beeckmans : a) Fiches des Juifs inscrits par IAssociation des Juifs en Belgique (Avenue Louise : Sibbekundige Opzoekingen / Bureau des Études raciques généalogiques («mariages mixtes»: AA 1314, n's 362-368). $\triangle$ Fichier d'alarme de l'Oberfeldkommandantur Brussel:
fiches des services allemands à Bruxelles (AA 500). 1 fichier. $\diamond$ Dossier d'enquête Isabelle Ponteville : Avenue Louise,

$\diamond$ Auditorat militaire auprès du Conseil de guerre permanent de Bruxelles. Exposé des faits dans l'affair AA 310 .

\section{Archives de la Ville de Bruxelles}

$\diamond$ Plan des caves Avenue Louise $\left(n^{\circ} 737.45 .74\right)$

$\Leftrightarrow$ Dossier réquisition allemande : Avenue Louise, $n^{\circ} 347$, pu consulter tout le dossier. Actuellement, on ne retrouve plus que les fiches des propriétaires de limmeuble du 347 de

$\diamond$ Dossier réquisition alliée : Avenue Louise, $n^{\circ} 347, n^{\circ} 4663$. Publications

$\diamond$ [Bloch, Alain]. [Biographie Henri Levy-Morelle], s.d. (non publié).

$\diamond$ Dartevelle, André \& Ponteville Isabelle (1996): Avenue Louise n ${ }^{\circ} 377$ Louiszalaan. Dans les caves de la Gestapo. In de .

$\diamond$ Gerhard,Paul \& Mallmann, Klaus-Michael (Hrsg.) und besetztes Europa, Darmstadt, Wissenschaftliche
und Buchgesellschaft.

$\triangle$ Saerens, Lieven (2007) : «La politique antijuive de AAllemagne nazie (1933-1945) », in Rudi Van Doorslaer (dir.), Emmanuel Debruyne, Frank Seberechts, Nico Wouters, avec la participation de Lieven Saerens, La Belgique docile. Les la Seconde Guerre mondiale, Bruxelles, CEGES, p. 21-46. $\diamond$ Saerens, Lieven (2014): Rachel, Jacob, Paul et les autres $\$$ Steinberg, Maxime \& Schram, Laurence (2009) : e.a,
Mechlen - Auschwitz 1942-1944, 4 volumes, Malines/ Bruxelles, VUBPRESS.

$\triangle$ Weyssow, Daniel (2008) : « L Le siège de la Gestapo Bruxelles. Un non-lieu de mémoire », Questions de
communication, série actes, $n^{\circ}$ 5, p. 195-203 (Actes du colloque «Qualifier des lieux de détention et de massacre des 8 au 10 novembre 2007).

$\diamond$ Weyssow, Daniel (dir.) (2014) : Les caves de la Gestapo. Reconnaissance et conservation, Paris, Kimé. 